\title{
A NEW CHARACTERIZATION OF STABLE NEURAL NETWORK CONTROL FOR DISCRETE-TIME UNCERTAIN SYSTEMS
}

\author{
Tomohisa Hayakawa ${ }^{*}$ Wassim M. Haddad ${ }^{* *}$ \\ Naira Hovakimyan ${ }^{* * *}$ \\ * CREST, Japan Science and Technology Agency, \\ Saitama, 332-0012, JAPAN \\ ** School of Aerospace Engineering, \\ Georgia Institute of Technology, Atlanta, GA 30332-0150, USA \\ *** Department of Aerospace and Ocean Engineering, \\ Virginia Polytechnic Institute and State University, \\ Blacksburg, VA 24061, USA
}

\begin{abstract}
A novel neuro adaptive control framework for discrete-time multivariable nonlinear uncertain systems is developed. The proposed framework is Lyapunov-based and guarantees, instead of ultimate boundedness, partial asymptotic stability of the closedloop system; that is, Lyapunov stability of the closed-loop system states and attraction with respect to the plant states. Unlike standard neural network approximation, we assume that the approximation error can be confined in a small gain-type norm-bounded conic sector over a compact set. This helps to couple tools from robust control with adaptive laws in discrete time to prove partial asymptotic stability of the closed-loop system. Finally, an illustrative numerical example is provided to demonstrate the efficacy of the proposed approach. Copyright (C) 2005 IFAC
\end{abstract}

Keywords: Adaptive control, neural network, discrete-time systems, asymptotic stability, sector-bounded nonlinearities, Lyapunov method

\section{INTRODUCTION}

Due to the severe complexities, nonlinearities, and uncertainties in modern controlled systems, neural networks provide an ideal framework for adaptive control because of their parallel processing flexibility and adaptability. This is owing to the fact that neural networks can approximate continuous nonlinear maps from collective action of very simple and autonomous process units that are mutually connected in simple ways. Consequently, use of the neural networks for system identification and control is one of the major areas of research interest.

Even though neural network-based adaptive control algorithms have been extensively developed in the lit- erature, it is quite common using Lyapunov-like functions to claim that the neural network controllers can guarantee ultimate boundedness of the closed-loop system states. This implies that the plant states converge to a neighborhood of an equilibrium point (see, for example, Chen \& Khalil (1995), Jagannathan \& Lewis (1996), Ge et al. (2003) for discrete-time cases). The reason why stability is not guaranteed stems from the fact that uncertainties in the system dynamics cannot be captured by neural networks perfectly and the residual approximation error is characterized via infinity norm over a given compact set. As one can surmise, however, the ultimate boundedness claims are somewhat conservative since standard Lyapunov-like theorems typically used to show ultimate boundedness of the closed-loop states provide only sufficient con- 
ditions, while neural network controllers may possibly achieve plant state convergence to the equilibrium point.

In this paper we develop a neuro adaptive control framework for a class of discrete-time nonlinear uncertain dynamical systems which ensures state convergence as well as boundedness of the neural network weighting gains. Specifically, the proposed framework is Lyapunov-based and guarantees partial asymptotic stability of the closed-loop system; that is, Lyapunov stability of the overall closed-loop states and convergence with respect to the plant state. The neuro adaptive controllers are constructed without requiring explicit knowledge of the system dynamics other than the fact that the plant dynamics are continuously differentiable and that the approximation error of unknown nonlinearities lies in a small gain-type norm bounded conic sector over a compact set. Furthermore, the proposed neuro control architecture is modular in the sense that if a nominal linear design model is available, the neuro adaptive controller can be augmented to the nominal design to account for system nonlinearities and system uncertainty.

Finally, we emphasize that we do not impose any linear growth condition on the system dynamics. Note that in the literature on classical adaptive control for discrete-time systems, it is typically assumed that the nonlinear system dynamics have the linear growth rate which is necessary in proving Lyapunov stability rather than practical stability (ultimate boundedness). Our novel characterization of system uncertainties (the small-gain type bound on the norm of the modelling error) allows to prove asymptotic stability without requiring linear growth condition for the system dynamics.

The notation used in this paper is fairly standard. Specifically, $\mathbb{R}$ denotes the set of real numbers, $\mathbb{R}^{n}$ denotes the set of $n \times 1$ real column vectors, and $\mathcal{N}$ denotes the set of nonnegative integers. Furthermore, we write $(\cdot)^{\mathrm{T}}$ to denote transpose, $\operatorname{tr}(\cdot)$ for the trace operator, $\ln (\cdot)$ for the natural $\log$ operator, $\sigma_{\max }(\cdot)$ to denote the maximum singular value of a matrix, $\operatorname{vec}(\cdot)$ denotes the column stacking operator for a matrix, and $\|\cdot\|$ for the Euclidean vector norm.

\section{STABLE NEURO ADAPTIVE CONTROL FOR DISCRETE-TIME NONLINEAR UNCERTAIN SYSTEMS}

In this section, we characterize neural adaptive feedback laws for discrete-time nonlinear uncertain systems. Specifically, consider the controlled nonlinear uncertain dynamical system $\mathcal{G}$ given by

$$
\begin{array}{r}
x(k+1)=f(x(k))+G(x(k)) u(k), \quad x(0)=x_{0}, \\
k \in \mathcal{N}, \quad(1)
\end{array}
$$

where $x(k) \in \mathbb{R}^{n}, k \in \mathcal{N}$, is the state vector, $u(k) \in$ $\mathbb{R}^{m}, k \in \mathcal{N}$, is the control input, $f: \mathbb{R}^{n} \rightarrow \mathbb{R}^{n}$ is continuously differentiable and satisfies $f(0)=0$, and $G: \mathbb{R}^{n} \rightarrow \mathbb{R}^{n \times m}$.

In this section, we assume that $f(\cdot)$ is an unknown function and $f(\cdot)$ and $G(\cdot)$ are given by

$$
\begin{aligned}
f(x) & =A x+\Delta f(x), \\
G(x) & =B G_{\mathrm{n}}(x),
\end{aligned}
$$

where $A \in \mathbb{R}^{n \times n}$ and $B \in \mathbb{R}^{n \times m}$ are known matrices, $G_{\mathrm{n}}: \mathbb{R}^{n} \rightarrow \mathbb{R}^{m \times m}$ is a known matrix function such that $\operatorname{det} G_{\mathrm{n}}(x) \neq 0, x \in \mathbb{R}^{n}$, and $\Delta f: \mathbb{R}^{n} \rightarrow \mathbb{R}^{n}$ is an uncertain function belonging to the uncertainty set $\mathcal{F}$ given by

$$
\begin{array}{r}
\mathcal{F}=\left\{\Delta f: \mathbb{R}^{n} \rightarrow \mathbb{R}^{n}: \Delta f(0)=0,\right. \\
\left.\Delta f(x)=B \delta(x), x \in \mathbb{R}^{n}\right\},
\end{array}
$$

where $\delta: \mathbb{R}^{n} \rightarrow \mathbb{R}^{m}$ is an uncertain continuously differentiable function such that $\delta(0)=0$. It is important to note that since $\delta(x)$ is continuously differentiable and $\delta(0)=0$, it follows that there exists a continuous matrix function $\Delta: \mathbb{R}^{n} \rightarrow \mathbb{R}^{m \times n}$ such that $\delta(x)=\Delta(x) x, x \in \mathbb{R}^{n}$. Furthermore, we assume that the continuous matrix function $\Delta(\cdot)$ can be approximated over a compact set $\mathcal{D}_{\mathrm{c}} \subset \mathbb{R}^{n}$ by a linear in the parameters neural network up to a desired accuracy so that

$$
\begin{array}{r}
\operatorname{col}_{i}(\Delta(x))=W_{i}^{\mathrm{T}} \sigma(x)+\varepsilon_{i}(x), \quad x \in \mathcal{D}_{\mathrm{c}}, \\
i=1, \cdots, n,
\end{array}
$$

where $\operatorname{col}_{i}(\Delta(\cdot))$ denotes the $i$ th column of $\Delta(\cdot)$, $W_{i}^{\mathrm{T}} \in \mathbb{R}^{m \times s}, i=1, \cdots, n$, are optimal unknown (constant) weights that minimize the approximation error over $\mathcal{D}_{\mathrm{c}}, \varepsilon_{i}: \mathbb{R}^{n} \rightarrow \mathbb{R}^{m}, i=1, \cdots, n$, are modeling errors such that $\sigma_{\max }(\Upsilon(x)) \leq \gamma^{-1}, x \in$ $\mathbb{R}^{n}$, where $\Upsilon(x) \triangleq\left[\varepsilon_{1}(x), \cdots, \varepsilon_{n}(x)\right]$ and $\gamma>0$, and $\sigma: \mathbb{R}^{n} \rightarrow \mathbb{R}^{s}$ is a given basis function such that $0 \leq \sigma(x) \leq 1, x \in \mathbb{R}^{n_{x}}$.

Next, defining

$$
\varphi(x) \triangleq \delta(x)-W^{\mathrm{T}}[x \otimes \sigma(x)],
$$

where $W^{\mathrm{T}} \triangleq\left[W_{1}^{\mathrm{T}}, \cdots, W_{n}^{\mathrm{T}}\right] \in \mathbb{R}^{m \times n s}$, it follows from (5) and Cauchy-Schwartz inequality that

$$
\begin{aligned}
\varphi^{\mathrm{T}}(x) \varphi(x) & =\left\|\Delta(x) x-W^{\mathrm{T}}(x \otimes \sigma(x))\right\|^{2} \\
& =\|\Delta(x) x-\Sigma(x) x\|^{2} \\
& =\|\Upsilon(x) x\|^{2} \\
& \leq \gamma^{-2} x^{\mathrm{T}} x, \quad x \in \mathcal{D}_{\mathrm{c}},
\end{aligned}
$$

where $\Sigma(x) \triangleq\left[W_{1}^{\mathrm{T}} \sigma(x), \cdots, W_{n}^{\mathrm{T}} \sigma(x)\right]$ and $\otimes$ denotes Kronecker product. This corresponds to a nonlinear small gain-type norm bounded uncertainty characterization for $\varphi(\cdot)$ (see Figure 1).

Theorem 2.1. Consider the nonlinear uncertain dynamical system $\mathcal{G}$ given by (1) where $f(\cdot)$ and $G(\cdot)$ are 


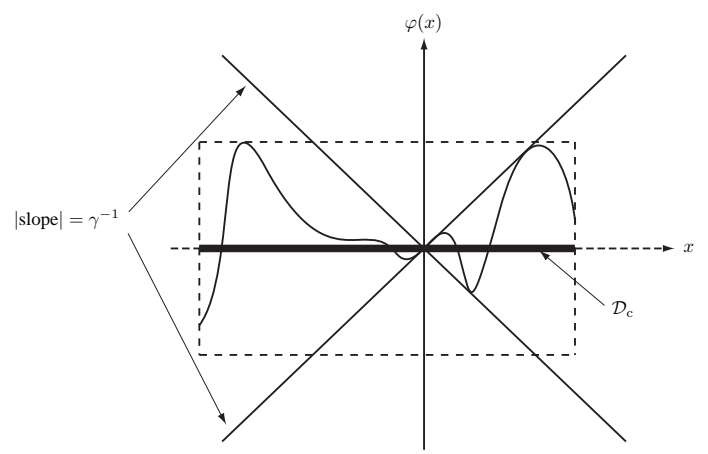

Fig. 1. Visualization of function $\varphi(\cdot)$

given by (2) and (3), respectively, and $\Delta f(\cdot)$ belongs to $\mathcal{F}$. Assume there exists a matrix $K \in \mathbb{R}^{m \times n}$ such that $A_{\mathrm{s}} \triangleq A+B K$ is asymptotically stable. Furthermore, for a given $\gamma>0$, assume there exist positivedefinite matrices $P \in \mathbb{R}^{n \times n}$ and $R \in \mathbb{R}^{n \times n}$ such that

$$
\begin{aligned}
P= & A_{\mathrm{s}}^{\mathrm{T}} P A_{\mathrm{s}}+A_{\mathrm{s}}^{\mathrm{T}} P B\left[\gamma^{2} I_{m}-B^{\mathrm{T}} P B\right]^{-1} B^{\mathrm{T}} P A_{\mathrm{s}} \\
& +(1+\alpha+\beta) I_{n}+R,
\end{aligned}
$$

where $\alpha>0, \gamma^{2} I_{m}-B^{\mathrm{T}} P B>0$, and, for all $x \in \mathcal{D}_{\mathrm{c}}$, $\beta$ satisfies

$$
\begin{aligned}
& \beta \geq \max \left\{c, n / \lambda_{\min }(P)\right\} \gamma^{-2} \frac{1+x^{\mathrm{T}} P x}{c+\tilde{\sigma}^{\mathrm{T}}(x) \tilde{\sigma}(x)} \\
& \cdot\left(\frac{2}{\alpha} B^{\mathrm{T}} P A_{\mathrm{s}} A_{\mathrm{s}}^{\mathrm{T}} P B+\frac{2}{\alpha \gamma^{2}}\left(B^{\mathrm{T}} P B\right)^{2}+B^{\mathrm{T}} P B\right),
\end{aligned}
$$

where $\tilde{\sigma}(x) \triangleq x \otimes \sigma(x)$ and $c>0$. Finally, let $c>0$. Then the neural adaptive feedback control law

$$
\begin{aligned}
u(k)= & G_{\mathrm{n}}^{-1}(x(k))[K x(k) \\
& \left.-\hat{W}^{\mathrm{T}}(k)[x(k) \otimes \sigma(x(k))]\right],
\end{aligned}
$$

where $\hat{W}^{\mathrm{T}}(k) \in \mathbb{R}^{m \times n s}, k \in \mathcal{N}$, and $\sigma: \mathbb{R}^{n} \rightarrow \mathbb{R}^{s}$ is a given basis function, with update law

$$
\begin{array}{r}
\hat{W}^{\mathrm{T}}(k+1)=\hat{W}^{\mathrm{T}}(k)+\frac{1}{c+\sigma^{\mathrm{T}}(x(k)) \sigma(x(k))\|x(k)\|^{2}} B^{\dagger} \\
\cdot\left[x(k+1)-A_{\mathrm{s}} x(k)\right][x(k) \otimes \sigma(x(k))]^{\mathrm{T}}, \\
\hat{W}^{\mathrm{T}}(0)=\hat{W}_{0}^{\mathrm{T}}, \quad(11)
\end{array}
$$

guarantees that there exists a positively invariant set $\mathcal{D}_{\alpha} \subset \mathbb{R}^{n} \times \mathbb{R}^{m \times n s}$ such that $\left(0, W^{\mathrm{T}}\right) \in \mathcal{D}_{\alpha}$, where $W^{\mathrm{T}} \in \mathbb{R}^{m \times n s}$, and the solution $\left(x(k), \hat{W}^{\mathrm{T}}(k)\right) \equiv$ $\left(0, W^{\mathrm{T}}\right)$ of the closed-loop system given by (1), (10), (11) is Lyapunov stable and $x(k) \rightarrow 0$ as $k \rightarrow \infty$ for all $\Delta f(\cdot) \in \mathcal{F}$ and $\left(x_{0}, \hat{W}_{0}^{\mathrm{T}}\right) \in \mathcal{D}_{\alpha}$.

Proof. First, note that with $u(k), k \in \mathcal{N}$, given by (10) it follows from (1)-(3) that

$$
\begin{array}{r}
x(k+1)=A x(k)+\Delta f(x(k))+B K x(k) \\
-B \hat{W}^{\mathrm{T}}(k)[x(k) \otimes \sigma(x(k))], \\
x(0)=x_{0}, \quad k \in \mathcal{N},
\end{array}
$$

or, equivalently, using (6),

$$
\begin{aligned}
& x(k+1)= A_{\mathrm{s}} x(k)+B[\varphi(x(k)) \\
&\left.-\tilde{W}^{\mathrm{T}}(k)[x(k) \otimes \sigma(x(k))]\right], \\
& x(0)=x_{0}, \quad k \in \mathcal{N},
\end{aligned}
$$

where $\tilde{W}^{\mathrm{T}}(k) \triangleq \hat{W}^{\mathrm{T}}(k)-W^{\mathrm{T}}$. Now, adding and subtracting $W^{\mathrm{T}}$ to and from (11) and using (13) it follows that

$$
\begin{aligned}
\tilde{W}^{\mathrm{T}}(k+1) & \\
= & \tilde{W}^{\mathrm{T}}(k)+\frac{1}{c+\sigma^{\mathrm{T}}(x(k)) \sigma(x(k))\|x(k)\|^{2}} B^{\dagger} B \\
& \cdot\left[\varphi(x(k))-\tilde{W}^{\mathrm{T}}(k) \tilde{\sigma}(x(k))\right] \tilde{\sigma}^{\mathrm{T}}(x(k)) \\
= & \tilde{W}^{\mathrm{T}}(k)+\frac{1}{c+\tilde{\sigma}^{\mathrm{T}}(x(k)) \tilde{\sigma}(x(k))}[\varphi(x(k)) \\
& \left.-\tilde{W}^{\mathrm{T}}(k) \tilde{\sigma}(x(k))\right] \tilde{\sigma}^{\mathrm{T}}(x(k)) .
\end{aligned}
$$

To show Lyapunov stability of the closed-loop system (11) and (13) consider the Lyapunov function candidate

$$
V\left(x, \tilde{W}^{\mathrm{T}}\right)=\ln \left(1+x^{\mathrm{T}} P x\right)+a \operatorname{tr} \tilde{W} \tilde{W}^{\mathrm{T}},
$$

where

$$
\begin{aligned}
a= & \max \left\{c, n / \lambda_{\min }(P)\right\}\left(\frac{2}{\alpha} B^{\mathrm{T}} P A_{\mathrm{s}} A_{\mathrm{s}}^{\mathrm{T}} P B\right. \\
& \left.+\frac{2}{\alpha \gamma^{2}}\left(B^{\mathrm{T}} P B\right)^{2}+B^{\mathrm{T}} P B\right) .
\end{aligned}
$$

Note that $V\left(0, \tilde{W}^{\mathrm{T}}\right)=0$ and, since $P$ is positive definite and $a>0, V\left(x, \tilde{W}^{\mathrm{T}}\right)>0$ for all $\left(x, \tilde{W}^{\mathrm{T}}\right) \neq$ $(0,0)$. Now, letting $x(k), k \in \mathcal{N}$, denote the solution to (13) and using (7), (8), and (11), it follows that the Lyapunov difference along the closed-loop system trajectories is given by

$$
\begin{aligned}
& \Delta V\left(x(k), \tilde{W}^{\mathrm{T}}(k)\right) \\
& \triangleq V\left(x(k+1), \tilde{W}^{\mathrm{T}}(k+1)\right)-V\left(x(k), \tilde{W}^{\mathrm{T}}(k)\right) \\
& =\ln \left(1+\left(A_{\mathrm{s}} x(k)+B[\varphi(x(k))\right.\right. \\
& \left.\left.-\tilde{W}^{\mathrm{T}}(k) \tilde{\sigma}(x(k))\right]\right)^{\mathrm{T}} P\left(A_{\mathrm{s}} x(k)+B[\varphi(x(k))\right. \\
& \left.\left.\left.-\tilde{W}^{\mathrm{T}}(k) \tilde{\sigma}(x(k))\right]\right)\right)+a \operatorname{tr}\left(\tilde{W}^{\mathrm{T}}(k)\right. \\
& +\frac{1}{c+\tilde{\sigma}^{\mathrm{T}}(x(k)) \tilde{\sigma}(x(k))}\left[\varphi(x(k))-\tilde{W}^{\mathrm{T}}(k) \tilde{\sigma}(x(k))\right] \\
& \text { - } \left.\tilde{\sigma}^{\mathrm{T}}(x(k))\right)^{\mathrm{T}}\left(\tilde{W}^{\mathrm{T}}(k)+\frac{1}{c+\tilde{\sigma}^{\mathrm{T}}(x(k)) \tilde{\sigma}(x(k))}\right. \\
& \left.\cdot\left[\varphi(x(k))-\tilde{W}^{\mathrm{T}}(k) \tilde{\sigma}(x(k))\right] \tilde{\sigma}^{\mathrm{T}}(x(k))\right) \\
& -\ln \left(1+x^{\mathrm{T}}(k) P x(k)\right)-a \operatorname{tr} \tilde{W}(k) \tilde{W}^{\mathrm{T}}(k) \\
& =\ln \left(1+\left[x^{\mathrm{T}}(k) A_{\mathrm{s}}^{\mathrm{T}} P A_{\mathrm{s}} x(k)\right.\right. \\
& +2 x^{\mathrm{T}}(k) A_{\mathrm{s}}^{\mathrm{T}} P B \varphi(x(k)) \\
& -2 x^{\mathrm{T}}(k) A_{\mathrm{s}}^{\mathrm{T}} P B \tilde{W}^{\mathrm{T}}(k) \tilde{\sigma}(x(k))
\end{aligned}
$$




$$
\begin{aligned}
& +\varphi^{\mathrm{T}}(x(k)) B P B \varphi(x(k)) \\
& -2 \varphi^{\mathrm{T}}(x(k)) B P B \tilde{W}^{\mathrm{T}}(k) \tilde{\sigma}(x(k)) \\
& +\tilde{\sigma}^{\mathrm{T}}(x(k)) \tilde{W}(k) B P B \tilde{W}^{\mathrm{T}}(k) \tilde{\sigma}(x(k)) \\
& \left.\left.-x^{\mathrm{T}}(k) P x(k)\right]\left[1+x^{\mathrm{T}}(k) P x(k)\right]^{-1}\right) \\
& +a \operatorname{tr} \tilde{W}(k) \tilde{W}^{\mathrm{T}}(k)+\frac{2 a}{c+\tilde{\sigma}^{\mathrm{T}}(x(k)) \tilde{\sigma}(x(k))} \\
& \cdot \operatorname{tr} W(k)\left[\varphi(x(k))-\tilde{W}^{\mathrm{T}}(k) \tilde{\sigma}(x(k))\right] \tilde{\sigma}^{\mathrm{T}}(x(k)) \\
& +\frac{a}{\left(c+\tilde{\sigma}^{\mathrm{T}}(x(k)) \tilde{\sigma}(x(k))\right)^{2}} \operatorname{tr} \tilde{\sigma}(x(k))\left[\varphi^{\mathrm{T}}(x(k))\right. \\
& \left.-\tilde{\sigma}^{\mathrm{T}}(x(k)) \tilde{W}(k)\right]\left[\varphi(x(k))-\tilde{W}^{\mathrm{T}}(k) \tilde{\sigma}(x(k))\right] \\
& \text { - } \tilde{\sigma}^{\mathrm{T}}(x(k))-a \operatorname{tr} \tilde{W}(k) \tilde{W}^{\mathrm{T}}(k) \\
& \leq\left[-x^{\mathrm{T}}(k)\left((1+\alpha+\beta) I_{n}+R\right.\right. \\
& \left.+A_{\mathrm{s}}^{\mathrm{T}} P B\left[\gamma^{2} I_{m}-B^{\mathrm{T}} P B\right]^{-1} B^{\mathrm{T}} P A_{\mathrm{s}}\right) x(k) \\
& +2 x^{\mathrm{T}}(k) A_{\mathrm{s}}^{\mathrm{T}} P B \varphi(x(k)) \\
& -2 x^{\mathrm{T}}(k) A_{\mathrm{s}}^{\mathrm{T}} P B \tilde{W}^{\mathrm{T}}(k) \tilde{\sigma}(x(k)) \\
& +\varphi^{\mathrm{T}}(x(k)) B^{\mathrm{T}} P B \varphi(x(k)) \\
& -2 \varphi^{\mathrm{T}}(x(k)) B^{\mathrm{T}} P B \tilde{W}^{\mathrm{T}}(k) \tilde{\sigma}(x(k)) \\
& \left.+\tilde{\sigma}^{\mathrm{T}}(x(k)) \tilde{W}(k) B^{\mathrm{T}} P B \tilde{W}^{\mathrm{T}}(k) \tilde{\sigma}(x(k))\right] \\
& \cdot\left[1+x^{\mathrm{T}}(k) P x(k)\right]^{-1}+\frac{2 a}{c+\tilde{\sigma}^{\mathrm{T}}(x(k)) \tilde{\sigma}(x(k))} \\
& \cdot \operatorname{tr} \tilde{W}(k)\left[\varphi(x(k))-\tilde{W}^{\mathrm{T}}(k) \tilde{\sigma}(x(k))\right] \tilde{\sigma}^{\mathrm{T}}(x(k)) \\
& +\frac{a}{c+\tilde{\sigma}^{\mathrm{T}}(x(k)) \tilde{\sigma}(x(k))} \operatorname{tr}\left[\varphi^{\mathrm{T}}(x(k))\right. \\
& \left.-\tilde{\sigma}^{\mathrm{T}}(x(k)) \tilde{W}(k)\right]\left[\varphi(x(k))-\tilde{W}^{\mathrm{T}}(k) \tilde{\sigma}(x(k))\right] \\
& \leq\left[-x^{\mathrm{T}}(k)\left((1+\alpha+\beta) I_{n}+R\right) x(k)\right. \\
& -x^{\mathrm{T}}(k) A_{\mathrm{s}}^{\mathrm{T}} P B\left[\gamma^{2} I_{m}-B^{\mathrm{T}} P B\right]^{-1} B^{\mathrm{T}} P A_{\mathrm{s}} x(k) \\
& +x^{\mathrm{T}}(k) A_{\mathrm{s}}^{\mathrm{T}} P B\left(\gamma^{2} I_{m}-B^{\mathrm{T}} P B\right)^{-1} B^{\mathrm{T}} P A_{\mathrm{s}} x(k) \\
& +\varphi^{\mathrm{T}}(x(k))\left(\gamma^{2} I_{m}-B^{\mathrm{T}} P B\right) \varphi(x(k)) \\
& -2 x^{\mathrm{T}}(k) A_{\mathrm{s}}^{\mathrm{T}} P B \tilde{W}^{\mathrm{T}}(k) \tilde{\sigma}(x(k)) \\
& +\varphi^{\mathrm{T}}(x(k)) B^{\mathrm{T}} P B \varphi(x(k)) \\
& -2 \varphi^{\mathrm{T}}(x(k)) B^{\mathrm{T}} P B \tilde{W}^{\mathrm{T}}(k) \tilde{\sigma}(x(k)) \\
& \left.+\tilde{\sigma}^{\mathrm{T}}(x(k)) \tilde{W}(k) B^{\mathrm{T}} P B \tilde{W}^{\mathrm{T}}(k) \tilde{\sigma}(x(k))\right] \\
& \cdot\left[1+x^{\mathrm{T}}(k) P x(k)\right]^{-1}+\frac{2 a}{c+\tilde{\sigma}^{\mathrm{T}}(x(k)) \tilde{\sigma}(x(k))} \\
& \cdot \operatorname{tr} \tilde{W}(k)\left[\varphi(x(k))-\tilde{W}^{\mathrm{T}}(k) \tilde{\sigma}(x(k))\right] \tilde{\sigma}^{\mathrm{T}}(x(k)) \\
& +\frac{a}{c+\tilde{\sigma}^{\mathrm{T}}(x(k)) \tilde{\sigma}(x(k))} \operatorname{tr}\left[\varphi^{\mathrm{T}}(x(k))\right. \\
& \left.-\tilde{\sigma}^{\mathrm{T}}(x(k)) \tilde{W}(k)\right]\left[\varphi(x(k))-\tilde{W}^{\mathrm{T}}(k) \tilde{\sigma}(x(k))\right],
\end{aligned}
$$

where in (17) we used $\ln a-\ln b=\ln \frac{a}{b}$ and $\ln (1+$ $c) \leq c$ for $a, b>0$ and $c \geq-1$, respectively, and $\frac{\tilde{\sigma}^{\mathrm{T}} \tilde{\sigma}}{c+\tilde{\sigma}^{\mathrm{T}} \tilde{\sigma}}<1$. Furthermore, note that $\tilde{\sigma}^{\mathrm{T}}(x) \tilde{\sigma}(x) \leq$ $n x^{\mathrm{T}} x$.

Now, defining $Q_{1} \triangleq \frac{2}{\alpha} B^{\mathrm{T}} P A_{\mathrm{s}} A_{\mathrm{s}}^{\mathrm{T}} P B$ and $Q_{2} \triangleq$ $\frac{2}{\alpha \gamma^{2}}\left(B^{\mathrm{T}} P B\right)^{2}$, it follows from (17) that

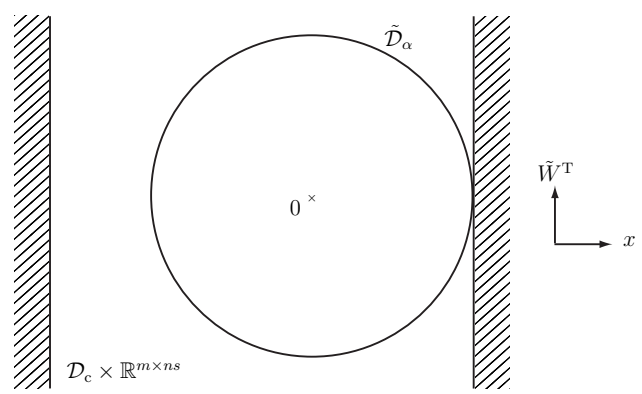

Fig. 2. Visualization of sets used in the proof of Theorem 2.1

$$
\begin{aligned}
\Delta V & \left(x(k), \tilde{W}^{\mathrm{T}}(k)\right) \\
\leq & {\left[-x^{\mathrm{T}}(k)\left(I_{n}+R\right) x(k)+x^{\mathrm{T}}(k) x(k)\right.} \\
& -\left[x^{\mathrm{T}}(k), \tilde{\sigma}^{\mathrm{T}}(x(k)) \tilde{W}(k)\right] \\
& \cdot\left[\begin{array}{cc}
\frac{1}{2} \alpha I_{n} & A_{\mathrm{s}}^{\mathrm{T}} P B \\
B^{\mathrm{T}} P A_{\mathrm{s}} & Q_{1}
\end{array}\right]\left[\begin{array}{c}
x(k) \\
\tilde{W}^{\mathrm{T}}(k) \tilde{\sigma}(x(k))
\end{array}\right] \\
& -\left[\varphi^{\mathrm{T}}(x(k)), \tilde{\sigma}^{\mathrm{T}}(x(k)) \tilde{W}(k)\right] \\
& \cdot\left[\begin{array}{ll}
\frac{1}{2} \alpha \gamma^{2} I_{n} & B^{\mathrm{T}} P B \\
B^{\mathrm{T}} P B & Q_{2}
\end{array}\right]\left[\begin{array}{c}
\varphi(x(k)) \\
\tilde{W}^{\mathrm{T}}(k) \tilde{\sigma}(x(k))
\end{array}\right] \\
& +\tilde{\sigma}^{\mathrm{T}}(x(k)) \tilde{W}(k)\left(Q_{1}+Q_{2}\right) \tilde{W}^{\mathrm{T}}(k) \tilde{\sigma}(x(k)) \\
& \left.+\tilde{\sigma}^{\mathrm{T}}(x(k)) \tilde{W}(k) B^{\mathrm{T}} P B \tilde{W}^{\mathrm{T}}(k) \tilde{\sigma}(x(k))\right] \\
& \cdot\left[1+x^{\mathrm{T}}(k) P x(k)\right]^{-1} \\
& -\frac{a}{c+\tilde{\sigma}^{\mathrm{T}}(x(k)) \tilde{\sigma}(x(k))} \tilde{\sigma}^{\mathrm{T}}(x(k)) \tilde{W}(k) \tilde{W}^{\mathrm{T}} \tilde{\sigma}(x(k)) \\
& +\frac{a}{c+\tilde{\sigma}^{\mathrm{T}}(x(k) \tilde{\sigma}(x(k))} \varphi^{\mathrm{T}}(x(k)) \varphi(x(k)) \\
\leq & -\frac{x^{\mathrm{T}}(k) R x(k)}{1+x^{\mathrm{T}}(k) P x(k)} \\
& -\frac{\tilde{\sigma}^{\mathrm{T}}(x(k)) \tilde{W}(k) \tilde{R}(x(k)) \tilde{W}^{\mathrm{T}}(k) \tilde{\sigma}(x(k))}{\left(c+\tilde{\sigma}^{\mathrm{T}}(x(k)) \tilde{\sigma}(x(k))\right)\left(1+x^{\mathrm{T}}(k) P x(k)\right)}
\end{aligned}
$$

where

$$
\begin{aligned}
\tilde{R}(x) \triangleq & a\left(1+x^{\mathrm{T}} P x\right) I_{m} \\
& -\left(Q_{1}+Q_{2}+B^{\mathrm{T}} P B\right)\left(c+\tilde{\sigma}^{\mathrm{T}}(x) \tilde{\sigma}(x)\right) \\
\geq & a\left(1+x^{\mathrm{T}} P x\right) I_{m} \\
& -\left(Q_{1}+Q_{2}+B^{\mathrm{T}} P B\right)\left(c+n x^{\mathrm{T}} x\right) \\
\geq & 0, \quad x \in \mathcal{D}_{c} .
\end{aligned}
$$

Hence, the Lyapunov difference given by (18) yields

$$
\begin{array}{r}
\Delta V\left(x(k), \tilde{W}^{\mathrm{T}}(k)\right) \leq-\frac{x^{\mathrm{T}}(k) R x(k)}{1+x^{\mathrm{T}}(k) P x(k)} \leq 0, \\
k \in \mathcal{N} .
\end{array}
$$

Next, let

$\tilde{\mathcal{D}}_{\alpha} \triangleq\left\{\left(x, \tilde{W}^{\mathrm{T}}\right) \in \mathbb{R}^{n} \times \mathbb{R}^{m \times n s}: V\left(x, \tilde{W}^{\mathrm{T}}\right) \leq \alpha\right\}$,

where $\alpha$ is the maximum value such that $\tilde{\mathcal{D}}_{\alpha} \subseteq \mathcal{D}_{\mathrm{c}} \times$ $\mathbb{R}^{m \times n s}$ (see Figure 2). Now, since $\Delta V\left(x(k), W^{\mathrm{T}}(k)\right)$ $\leq 0$ for all $\left(x(k), \tilde{W}^{\mathrm{T}}(k)\right) \in \tilde{\mathcal{D}}_{\alpha}$ and $k \in \mathcal{N}$, it 
follows that $\tilde{\mathcal{D}}_{\alpha}$ is positively invariant. Next, since $\tilde{\mathcal{D}}_{\alpha}$ is positively invariant, it follows that

$$
\begin{gathered}
\mathcal{D}_{\alpha} \triangleq\left\{\left(x, \hat{W}^{\mathrm{T}}\right) \in \mathbb{R}^{n} \times \mathbb{R}^{m \times n s}:\right. \\
\left.\left(x, \hat{W}^{\mathrm{T}}-W^{\mathrm{T}}\right) \in \tilde{\mathcal{D}}_{\alpha}\right\}
\end{gathered}
$$

is also positively invariant. Furthermore, it follows from (20) and (the discrete version of) Theorem 2 of (Chellaboina \& Haddad 2002) that the solution $\left(x(k), \hat{W}^{\mathrm{T}}(k)\right) \equiv\left(0, W^{\mathrm{T}}\right)$ to (11) and (13) is Lyapunov stable and $x(k) \rightarrow 0$ as $k \rightarrow \infty$ for all $\Delta f(\cdot) \in \mathcal{F}$ and $\left(x_{0}, \hat{W}_{0}\right) \in \mathcal{D}_{\alpha}$.

Remark 2.1. The conditions in Theorem 2.1 imply partial asymptotic stability, that is, the solution $(x(k)$, $\left.\hat{W}^{\mathrm{T}}(k)\right) \equiv\left(0, W^{\mathrm{T}}\right)$ of the overall closed-loop system is Lyapunov stable and $x(k) \rightarrow 0$ as $k \rightarrow \infty$. Hence, it follows from $(11)$ that $\hat{W}^{\mathrm{T}}(k+1)-\hat{W}^{\mathrm{T}}(k) \rightarrow 0$ as $k \rightarrow \infty$.

Remark 2.2. Since the Lyapunov function $V\left(x, \tilde{W}^{\mathrm{T}}\right)$ used in the proof of Theorem 2.1 is radially unbounded, the control law (10) ensures global asymptotic stability in the case where the neural network approximation holds in $\mathbb{R}^{n}$. However, the existence of a global neural network approximator for an uncertain nonlinear map cannot in general be established. Hence, as is common in the neural network literature, for a given arbitrarily large compact set $\mathcal{D}_{\mathrm{c}} \subset \mathbb{R}^{n}$, we assume that there exists an approximator for the unknown nonlinear map up to a desired accuracy. In the case where $\Delta(\cdot)$ is continuous on $\mathbb{R}^{n}$, it follows from the Stone-Weierstrass theorem that $\Delta(\cdot)$ can be approximated over an arbitrarily large compact set $\mathcal{D}_{\mathrm{c}}$. In this case, our neuro adaptive controller guarantees semiglobal partial asymptotic stability.

Remark 2.3. Note that the neuro adaptive controller (10) and (11) can be constructed to guarantee partial asymptotic stability using standard discrete-time linear $\mathrm{H}_{\infty}$ theory. Specifically, it follows from standard discrete-time $\mathrm{H}_{\infty}$ theory $(\mathrm{Gu}$ et al. 1989) that $\|G(z)\|_{\infty}<\gamma$, where $G(z) \sim\left[\begin{array}{c|c}A_{\mathrm{s}} & B \\ \hline E & 0\end{array}\right]$ and $E$ is such that $E^{\mathrm{T}} E=(1+\alpha+\beta) I_{n}+R$, if and only if there exists a positive-definite matrix $P$ satisfying the discrete-time bounded real Riccati equation (8).

It is important to note that the adaptive control law (10) and (11) does not require the explicit knowledge of the optimal weighting matrix $W$. Furthermore, no specific structure on the nonlinear dynamics $f(x)$ is required to apply Theorem 2.1 . However, if (1) is in normal form (Isidori 1995), then we can always construct a neuro adaptive control law without requiring knowledge of the system dynamics $f(x)$. To see this, assume that the nonlinear uncertain system $\mathcal{G}$ is generated by the difference model

$$
\begin{array}{r}
z_{i}\left(k+\tau_{i}\right)=f_{\mathrm{u}_{i}}(z(k))+\sum_{j=1}^{m} G_{\mathrm{s}(i, j)}(z(k)) u_{j}(k), \\
k \in \mathcal{N}, \quad i=1, \cdots, m,
\end{array}
$$

where $\tau_{i} \in \mathcal{N}$ denotes the time delay (or relative degree) with respect to the output $z_{i}, z(k)=$ $\left[z_{1}(k), \cdots, z_{1}\left(k+\tau_{1}-1\right), \cdots, z_{m}(k), \cdots, z_{m}(k+\right.$ $\left.\left.\tau_{m}-1\right)\right]$, and $z(0)=z_{0}$. Here, we assume that the square matrix function $G_{\mathrm{S}}(z)$ composed of the entries $G_{\mathrm{s}(i, j)}(z), i, j=1, \cdots, m$, is such that $\operatorname{det} G_{\mathrm{s}}(z) \neq$ $0, z \in \mathbb{R}^{\hat{\tau}}$, where $\hat{\tau}=\tau_{1}+\cdots+\tau_{m}$. Furthermore, since (23) is in a form where it does not possess internal dynamics, it follows that $\hat{\tau}=n$. The case where (23) possesses input-to-state stable internal dynamics can be analogously handled as shown in Hayakawa et al. (2004).

Next, define $x_{i}(k) \triangleq\left[z_{i}(k), \cdots, z_{i}\left(k+\tau_{i}-2\right)\right]^{\mathrm{T}}, i=$ $1, \cdots, m, x_{m+1}(k) \triangleq\left[z_{1}\left(k+\tau_{1}-1\right), \cdots, z_{m}(k+\right.$ $\left.\left.\tau_{m}-1\right)\right]^{\mathrm{T}}$, and $x(k) \triangleq\left[x_{1}^{\mathrm{T}}(k), \cdots, x_{m+1}^{\mathrm{T}}(k)\right]^{\mathrm{T}}$ so that (23) can be described by (1) with

$$
\begin{aligned}
A & =\left[\begin{array}{c}
A_{0} \\
0_{m \times n}
\end{array}\right], \quad \Delta f(x)=\left[\begin{array}{c}
0_{(n-m) \times 1} \\
f_{\mathrm{u}}(x)
\end{array}\right], \\
G(x) & =\left[\begin{array}{c}
0_{(n-m) \times m} \\
G_{\mathrm{S}}(x)
\end{array}\right],
\end{aligned}
$$

where $A_{0} \in \mathbb{R}^{(n-m) \times n}$ is a known matrix of zeros and ones capturing the multivariable controllable canonical form representation (Chen 1984), $f_{\mathrm{u}}: \mathbb{R}^{n} \rightarrow \mathbb{R}^{m}$ is an unknown function and satisfies $f_{\mathrm{u}}(0)=0$, and $G_{\mathrm{S}}: \mathbb{R}^{n} \rightarrow \mathbb{R}^{m \times m}$. Note that $\Delta f(\cdot)$ belongs to $\mathcal{F}$ with $B=\left[0_{m \times(n-m)}, I_{m}\right]^{\mathrm{T}}$ and $\delta(x)=f_{\mathrm{u}}(x)$. In this case, $G_{\mathrm{n}}(x) \equiv G_{\mathrm{S}}(x)$. Furthermore, since $A$ is in multivariable controllable canonical form, we can always construct $K$ such that $A+B K$ is asymptotically stable.

\section{ILLUSTRATIVE NUMERICAL EXAMPLE}

In this section we present a numerical example to demonstrate the utility of the proposed discrete-time neural network adaptive control framework for adaptive stabilization. Specifically, consider the linear uncertain system given by

$$
\begin{array}{r}
z(k+2)+f_{\mathrm{u}}(z(k), z(k+1))=b u(k), \\
z(0)=z_{0}, \quad z(1)=z_{1}, \quad k \in \mathcal{N},
\end{array}
$$

where $z(k) \in \mathbb{R}, k \in \mathcal{N}, u(k) \in \mathbb{R}, k \in \mathcal{N}$, and $f_{\mathrm{u}}: \mathbb{R} \times \mathbb{R} \rightarrow \mathbb{R}$ is continuously differentiable. Note that with $x_{1}(k)=z(k)$ and $x_{2}(k)=$ $z(k+1)$, (25) can be written in state space form (1) with $x=\left[x_{1}, x_{2}\right]^{\mathrm{T}}, A=\left[\begin{array}{cc}0 & 1 \\ a_{1} & a_{2}\end{array}\right], \Delta f(x)=$ $\left[0,-a_{1} x_{1}-a_{2} x_{2}-f_{\mathrm{u}}\left(x_{1}, x_{2}\right)\right]^{\mathrm{T}}$, and $G(x)=[0, b]^{\mathrm{T}}$, where $a_{1}, a_{2} \in \mathbb{R}$. Here, we assume that $\Delta f(x)$ is unknown and can be written as $\Delta f(x)=B \delta(x)$, where $\delta(x)=-\frac{1}{b}\left[a_{1} x_{1}+a_{2} x_{2}+f_{\mathrm{u}}\left(x_{1}, x_{2}\right)\right]$ is an 

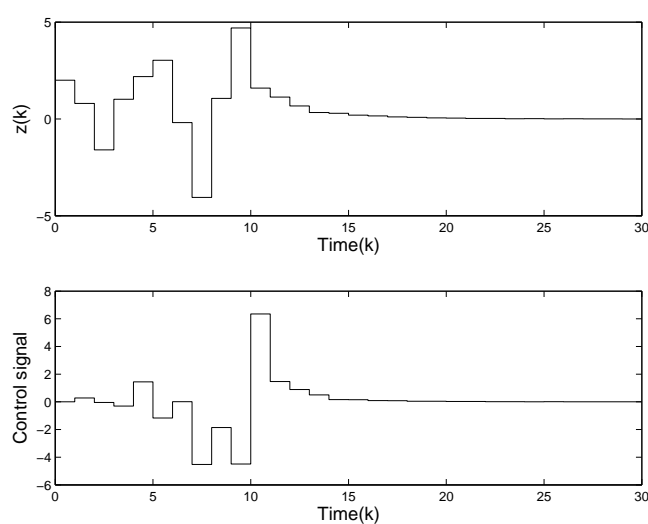

Fig. 3. State trajectory and control signal versus time unknown, continuously differentiable function. Next, let $K=\frac{1}{b}\left[k_{1}, k_{2}\right]$, where $k_{1}, k_{2}$ are arbitrary scalars, so that $A_{\mathrm{s}}=A+B K=\left[\begin{array}{cc}0 & 1 \\ a_{1}+k_{1} & a_{2}+k_{2}\end{array}\right]$. Now, with the proper choice of $k_{1}$ and $k_{2}$, it follows from Theorem 2.1 that if there exists $P>0$ satisfying (8), then the adaptive feedback controller (10) guarantees that $x(k) \rightarrow 0$ as $k \rightarrow \infty$. Specifically, here we choose $a_{1}=0, a_{2}=0, k_{1}=0.1, k_{2}=0.1, b=1$, $c=1, \gamma=18, \alpha=1, \beta=2.8001, \sigma(x)=$ $\left[1, \tanh \left(\lambda_{1} x_{1}\right), \cdots, \tanh \left(6 \lambda_{1} x_{1}\right), \tanh \left(\lambda_{2} x_{2}\right), \cdots\right.$, $\left.\tanh \left(6 \lambda_{2} x_{2}\right)\right]^{\mathrm{T}}$, where $\lambda_{1}=\lambda_{2}=0.1$, and $R=$ $0.1999 I_{2}$ so that $P$ satisfying (8) is given by

$$
P=\left[\begin{array}{rr}
5.1057 & 0.1179 \\
0.1179 & 10.2358
\end{array}\right]
$$

With $f_{\mathrm{u}}\left(x_{1}, x_{2}\right)=c_{1} \frac{x_{1}^{3}}{1+x_{1}^{2}}+c_{2} \ln \left(1+x_{2}^{2}\right)+c_{3} x_{1}^{2}, c_{1}=$ $1.5, c_{2}=-0.8, c_{3}=-0.2$, and initial conditions $x(0)=[2,0.8]^{\mathrm{T}}$ and $\hat{W}(0)=0_{26 \times 1}$, Figure 3 shows the state trajectory versus time and the control signal versus time. Finally, Figure 4 shows the adaptive gain history versus time.

\section{CONCLUSION}

A neuro adaptive control framework for adaptive stabilization of discrete-time multivariable nonlinear uncertain systems was developed. Using Lyapunov methods along with the standard robust control theory the proposed framework was shown to guarantee partial asymptotic stability of the closed-loop system rather than ultimate boundedness; that is, asymptotic stability with respect to part of the closed-loop system states associated with the plant. Finally, an illustrative numerical example was presented to show the utility of the proposed neuro adaptive stabilization scheme.

\section{ACKNOWLEDGEMENT}

This research was supported in part by the Air Force Office of Scientific Research under Grants F4962003-1-0178 and F49620-03-1-0443.
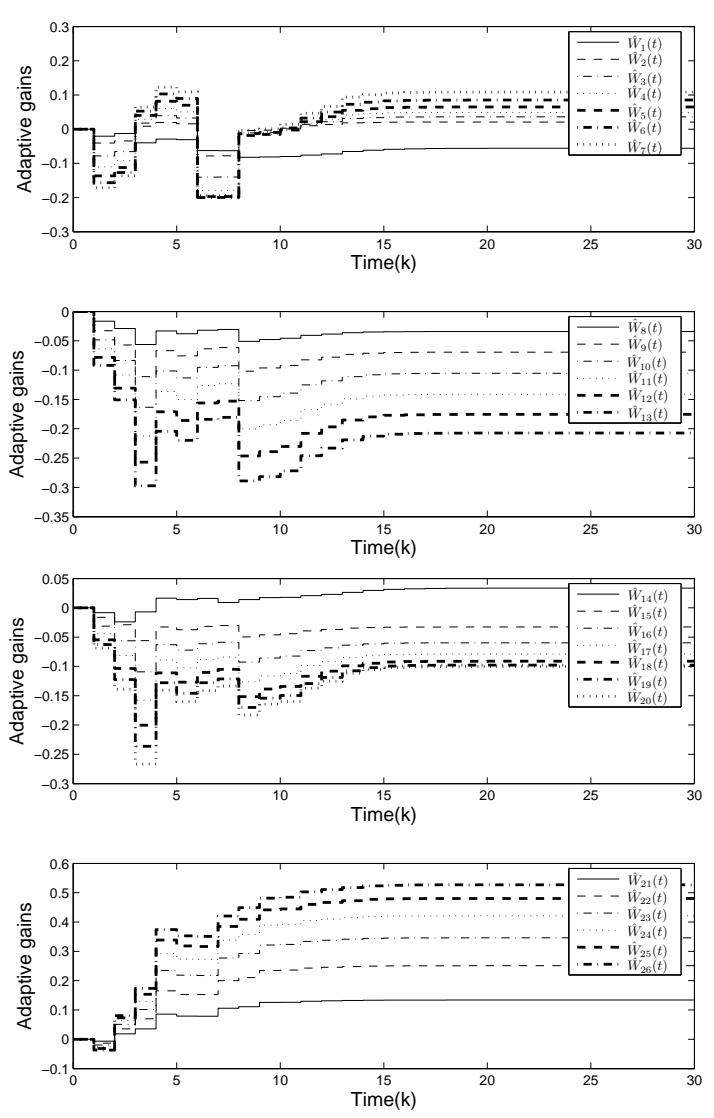

Fig. 4. Neural network weighting functions versus time

\section{REFERENCES}

Chellaboina, V. \& Haddad, W. M. (2002). A unification between partial stability and stability theory for time-varying systems. Control Systems Magazine, 22, 66-75.

Chen, C.-T. (1984). Linear System Theory and Design. New York: Holt, Rinehart, and Winston.

Chen, F. C. \& Khalil, H. K. (1995). Adaptive control of a class of nonlinear discrete-time systems using neural networks. IEEE Transactions on Automatic Control, 40(5), 791-801.

Ge, S. S., Lee, T. H., Li, G. Y. \& Zhang, J. (2003). Adaptive NN control for a class of discretetime non-linear systems. International Journal of Control, 76(4), 334-354.

Gu, D. W., Tsai, M. C., O'Young, S. D. \& Postlethwaite, I. (1989). State-space formulae for discrete-time $\mathrm{H}_{\infty}$ optimization. International Journal of Control, 49, 1683-1723.

Hayakawa, T., Haddad, W. M. \& Leonessa, A. (2004). A Lyapunov-based adaptive control framework for discrete-time nonlinear systems with exogenous disturbances. International Journal of Control, 77, 250-263.

Isidori, A. (1995). Nonlinear Control Systems. New York, NY: Springer.

Jagannathan, S. \& Lewis, F. L. (1996). Discrete-time neural net controller for a class of nonlinear dynamical systems. IEEE Transactions on Automatic Control, 41(11), 1693-1699. 